\title{
Computerised Methodologies for Non-Invasive Angiography-Derived Fractional Flow Reserve Assessment: A Critical Review
}

\author{
Anantharaman Ramasamy, ${ }^{1,2}$ Chongying Jin, ${ }^{1}$ Vincenzo Tufaro, ${ }^{1}$ Retesh Bajaj, ${ }^{1,2}$ \\ Yakup Kilic, ${ }^{1}$ Hannah Safi, ${ }^{1,3}$ Rajiv Amersey, ${ }^{1}$ Daniel Jones, ${ }^{1,2}$ Ryo Torii, ${ }^{3}$ \\ Alexandra Lansky, ${ }^{1,4}$ Anthony Mathur, ${ }^{1,2}$ Christos V. Bourantas, ${ }^{1,2,5}$ \\ and Andreas Baumbach (iD) \\ ${ }^{1}$ Department of Cardiology, Barts Heart Centre, Barts Health NHS Trust, London, UK \\ ${ }^{2}$ William Harvey Research Institute, Queen Mary University London, UK \\ ${ }^{3}$ Department of Mechanical Engineering, University College London, London, UK \\ ${ }^{4}$ Division of Cardiovascular Medicine, Department of Internal Medicine, Yale School of Medicine, New Haven, CT, USA \\ ${ }^{5}$ Institute of Cardiovascular Sciences, University College London, London, UK
}

Correspondence should be addressed to Andreas Baumbach; a.baumbach@qmul.ac.uk

Received 1 December 2019; Accepted 21 February 2020; Published 20 April 2020

Academic Editor: David G. Iosseliani

\begin{abstract}
Copyright (c) 2020 Anantharaman Ramasamy et al. This is an open access article distributed under the Creative Commons Attribution License, which permits unrestricted use, distribution, and reproduction in any medium, provided the original work is properly cited.
\end{abstract}

\begin{abstract}
Fractional flow reserve is the gold standard for assessing the haemodynamic significance of intermediate coronary artery stenoses. Cumulative evidence has shown that FFR-guided revascularisation reduces stent implantations and improves patient outcomes. However, despite the wealth of evidence and guideline recommendations, its use in clinical practice remains minimal. Patient and technical limitations of FFR as well as the need for intracoronary instrumentation, use of adenosine, and increased costs have limited FFR's applicability in clinical practice. Over the last decade, several angiography-derived FFR software packages have been developed which do not require intracoronary pressure assessment with a guidewire or need for administration of hyperaemic agents. At present, there are 3 commercially available software packages and several other non-commercial technologies that have been described in the literature. These technologies have been validated against invasive FFR showing good accuracy and correlation. However, the methodology behind these solutions is different-some algorithms are based on solving the governing equations of fluid dynamics such as the Navier-Stokes equation while others have opted for a more simplified mathematical formula approach. The aim of this review is to critically appraise the methodology behind all the known angiography-derived FFR technologies highlighting the key differences and limitations.
\end{abstract}

\section{Introduction}

Coronary angiography remains the established method for assessing the presence and severity of coronary artery disease. However, when an intermediate lesion (defined as diameter stenosis $40-90 \%$ ) is identified on coronary angiography, further evaluation of its clinical significance is advised [1]. This is due to the discrepancy between anatomical narrowing and functional effect on the flow reserve in intermediate coronary stenosis [2, 3]. Andreas Gruentzig, the pioneer of coronary angioplasty, showed that post-angioplasty reduction in pressure across the stenotic lesion is a useful indicator of a successful procedure [4]. Today, fractional flow reserve (FFR) is regarded as the gold standard for evaluating the functional significance of intermediate lesions and guiding revascularisation [5]. There are robust data from multiple randomised controlled trials that support the use of FFR to guide revascularisation resulting in reduced major 
adverse cardiac events, improved patient outcomes, reduced stent implantation rates, and cost-effectiveness [6-8]. However, the use of FFR in clinical practice remains limited due to the invasive nature of the assessment, which has an increase in complication rates, and albeit small, often produces symptoms from the hyperaemic agents, as well as an increase in procedural time and associated costs.

One of the main limitations of two-dimensional (2D) coronary angiography is the presence of foreshortening and the difficulty in accurately assessing diffuse long and eccentric lesions. The introduction of 3D quantitative coronary angiography (3D-QCA), which combines two projections, addresses some of these limitations and has shown a stronger correlation with invasive functional assessment of coronary stenoses $[9,10]$. More importantly, 3D-QCA also allows reconstruction of specific coronary geometries, which can then processed by computational methodologies that allow assessment of translesional pressure gradients.

Over the last decade, several angiography-derived methodologies that use 3D-QCA anatomic parameters to estimate FFR have been developed. These incorporate either blood flow simulation using computational fluid dynamic (CFD) techniques or a mathematical formula, which provides rapid calculation of the pressure drop across a lesion. The aim of this review is to critically appraise all the currently known angiography-derived FFR technologies in chronological order, focussing on their advantages and limitations.

\section{Angiography-Derived FFR Software}

2.1. Virtual Fractional Flow Reserve ( $v F F R)$. The pioneering study of angiography-derived FFR was by Morris et al. [11] in 2011. The VIRTU-1 study included 35 lesions from 19 patients and examined the feasibility of computational workflow based on coronary angiography to predict FFR. The successful application of computational fluid dynamics techniques to computed tomographic coronary angiography (CTCA) images to calculate lesion-specific FFR and the superior resolution of coronary angiography encouraged the authors to focus on the application of CFD to create the first angiography-derived FFR software, the vFFR.

The methodology involves using 2D images from rotational coronary angiography to identify two projections from a similar phase of the cardiac cycle and that are at least $90^{\circ}$ apart, which are reconstructed using the Phillips 3D workstation. The $3 \mathrm{D}$ reconstructed vessel is exported as a virtual reality modelling language file into a workflow. The inlet and outlets are defined and capped, and the surface is meshed into approximately 1 million tetrahedral elements. The proximal boundary condition is defined as the average proximal transient pressure waveform, and CFD analysis is performed using the commercially available software (ANSYS CFX). The solver is based on the Navier-Stokes continuity equation and principles of momentum conservation. The downstream boundary condition is developed by averaging the resistance and compliance values from all patients included in the study which is then applied as a generic condition according to the Windkessel model [12].
The simulation output is then used to derive vFFR results. The main limitations of vFFR include the use of rotational angiography which is not widely used in the clinical arena, the use of generic boundary conditions and the use of pulsatile CFD which translates into a very long computational times ( $>24$ hours).

A few years later, the same group developed a novel "pseudotransient" analysis protocol for computing vFFR [13]. "Pseudotransient" refers to transient CFD results being approximated without the need to perform the time-consuming, fully transient analysis. The $3 \mathrm{D}$ reconstruction is similar to the previous vFFR simulation where the images are based on rotational coronary angiography. The CFD analysis is based on 2 steady-state analyses, which is used to derive linear and quadratic terms to characterise pressure and flow which are then used to derive vFFR. This new approach results in a significant improvement in the computation time from $>24$ hours to $<4$ minutes. The linear and quadratic equations used 9 parameters including coronary microvasculature resistance and compliance.

In 2019, the same group developed an extension to their vFFR software, the Virtual Coronary Intervention (VCI) [14]. The 3D reconstruction is based on 2 orthogonal views, as close to $90^{\circ}$ from routine coronary angiography. Enddiastolic frames are automatically chosen based on the electrocardiographic tracing. The 3D model is then exported to the VIRTUheart workflow software. The surface is meshed based on the previous vFFR algorithm. The inlet boundary is set as the mean aortic pressure taken during angiography, and a generic resistance value of $8.721 E+9 \mathrm{~Pa} /$ $\mathrm{m}^{2} \mathrm{~s}^{-1}$ derived from the previous vFFR study is used to define the outlet boundary condition. Steady-state CFD computation is then performed using the commercially available (ANSYS CFX) software where the vFFR value can be calculated within a few minutes. The VCI allows virtual simulation of coronary stenting as part of the VIRTUheart software based on pre- and post-simulated vFFR calculation and prediction of physiological response to stenting.

\subsection{Virtual Functional Assessment Index (vFAI). After the} introduction of vFFR, Papafaklis et al. [15] introduced a new angiography-derived FFR (Figure 1). The vFAI's methodology is based on CFD. The first stage of calculating vFAI is based on accurate $3 \mathrm{D}-\mathrm{QCA}$ reconstruction using the validated CAAS 3D-QCA software (Pie Medical Imaging, Maastricht, the Netherlands). Pressure drop is linked to flow using linear and quadratic terms $\left(\Delta P=f_{\mathrm{v}} Q+f_{\mathrm{s}} Q^{2}\right)$, where $\Delta P$ is the pressure gradient $(\mathrm{mmHg}), Q$ is the flow rate $(\mathrm{ml} / \mathrm{s}), f_{\mathrm{v}}$ is the coefficient of pressure loss due to viscous friction, and $f_{\mathrm{s}}$ is the coefficient of pressure loss due to flow separation. The 3D-QCA geometry is used to perform two separate CFD simulations to solve the $f_{\mathrm{v}}$ and $f_{\mathrm{s}}$ parameters. The arterial wall is considered rigid, and no-slip conditions are applied at the vessel wall with a reference pressure of $100 \mathrm{mmHg}$ set at the inlet.

The vFAI does not require patient-specific blood flow measurements. The vFAI is calculated as the ratio of the area under curve for flow range between 0 and $4 \mathrm{mls} / \mathrm{sec}$ and is not identical to invasive FFR values. 


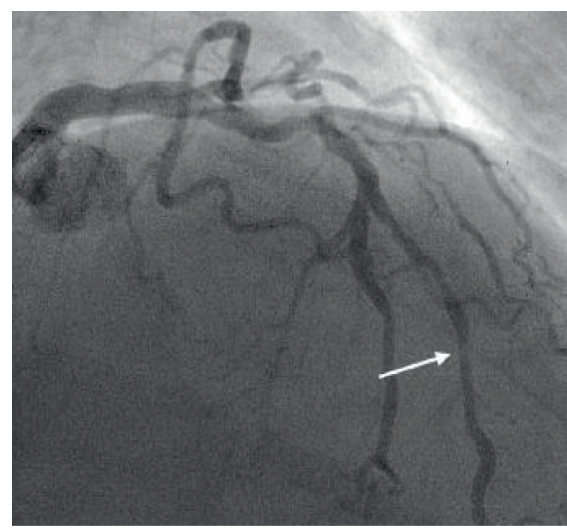

(a)

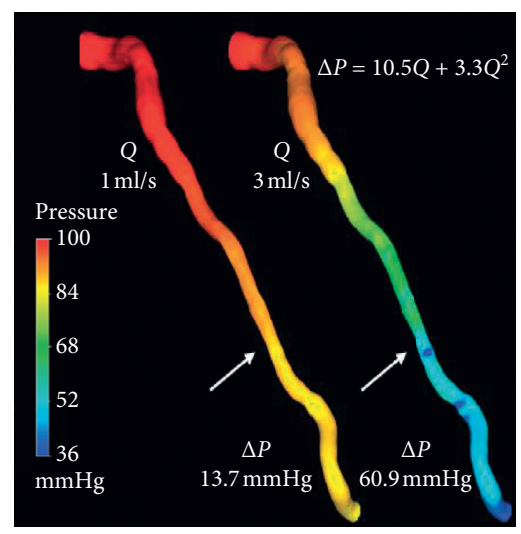

(b)

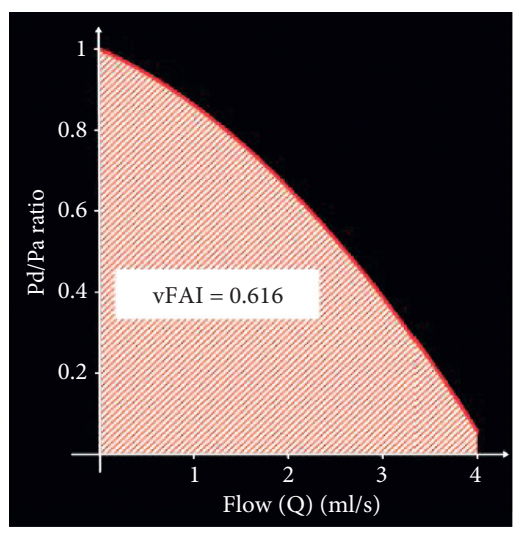

(c)

FiguRe 1: Functionally significant moderate lesion assessed by vFAI. (a) Coronary angiography image of a left anterior descending artery with an intermediate stenosis. The area with maximal diameter stenosis is marked with a white arrow. The haemodynamic significance of this lesion was assessed with invasive pressure wire showing FFR $=0.64$ (haemodynamically significant). (b) The 3D-QCA reconstruction model and colour-coded pressure distribution map at two different flow rates, $1 \mathrm{ml} / \mathrm{s}$ and $3 \mathrm{ml} / \mathrm{s}$. (c) Relationship between Pd/Pa ratio and coronary flow in the interrogated segment of the studied artery (reproduced with permission from Papafaklis et al. [15]).

The main limitations of vFAI are the exclusion of side branches and the assumption that coronary flow remains fixed across the length of the vessel. The average time required from extraction of 3D anatomy to completion of CFD is 15 minutes. The first prototype did not appear to be very user-friendly, and no further developments have been made since its introduction in 2014.

\subsection{Quantitative Flow Reserve (QFR), Medis Medical Imaging} System, Leiden, The Netherlands. QFR is an angiographyderived translesional physiology assessment software that was introduced after VFR and vFAI (Figure 2). The initial study by Tu et al. in 2014 was based on a CFD approach [16]. This study (where the computed FFR is termed FFR $_{\mathrm{QCA}}$ ) included 77 vessels from 68 patients and showed good correlation $(r=0.81, p<0.001)$ with invasive FFR. Furthermore, FFR $_{\mathrm{OCA}}$ showed a superior diagnostic accuracy to invasive FFR compared to minimum lumen area and diameter stenosis. Coronary angiography images were acquired at 15 or 30 frames/second; 3D-QCA was performed using the well-validated QAngioXA 3D software, and side branches with diameters larger than one-third of the main vessel were included in the reconstruction. When bifurcation lesions are present, the software reconstructs normal lumen borders, assuming there is no stenosis to determine the flow distribution between the main vessel and side branch. Multiple bifurcations in the same vessel are merged into a tree structure. The mean volumetric flow rate is calculated using the lumen volume of the reconstructed vessel divided by mean transport time, determined by thrombolysis in myocardial infarction (TIMI) frame count on hyperaemic acquisition obtained after adenosine infusion. The 3D geometry is meshed into tetrahedral cells, followed by the application of Navier-Stokes equations and non-linear partial differential equations (ANSYS Inc.). Blood is modelled as an incompressible and Newtonian fluid. Blood viscosity and density are derived from individual patient haematocrit information. Following the steady-state simulation, the FFR is calculated by dividing mean pressure at the outlet by mean pressure at the inlet.

Subsequent QFR studies have focussed on faster computation by adopting a mathematical approach [17]. Traditionally this was performed offline, although the workflow software can now be installed in the Cardiac Catheterisation Suite where angiographic images can be sent from the scanner to the workstation for fast analysis. The first step is similar to the earlier process, which involves creating a $3 \mathrm{D}$ reconstruction of the vessel using two angiographic projections, at least $25^{\circ}$ apart with minimal foreshortening and good vessel opacification. The side branches are not reconstructed in this approach. The QFR software computation works on the following principles: (1) coronary pressure remains constant through normal coronary arteries; (2) a simple quadratic equation using coefficients derived from flow data is used to calculate the pressure drop across a stenosis based on the geometry and flow; (3) 3D-QCA is able to accurately characterise stenosis and vessel geometry; and (4) coronary flow velocity distal to the stenosis is similar to that proximal to the stenosis and the mass flow rate at each location along the vessel can be calculated using the mean flow velocity and 3D-QCA anatomic parameters. The QFR software provides 3 different computational values depending on the mean hyperaemic flow velocities used. The first is fixed-QFR (fQFR) where a fixed hyperaemic flow velocity of $0.35 \mathrm{~m} / \mathrm{s}$ is used based on previous studies [16]. The second is contrast-QFR (cQFR) where frame count analysis on angiographic images without pharmacologically induced hyperaemia is used to calculate a flow velocity at nonhyperaemic conditions, which is then used to derive cQFR values. Finally, adenosine-QFR (aQFR) is similar to cQFR except that the angiographic projections used are acquired during hyperaemia (intravenous administration of adenosine) to provide the real hyperaemia flow velocity for 


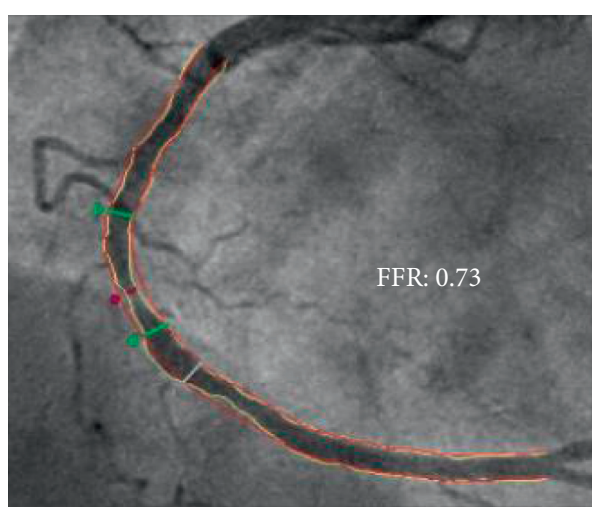

(a)

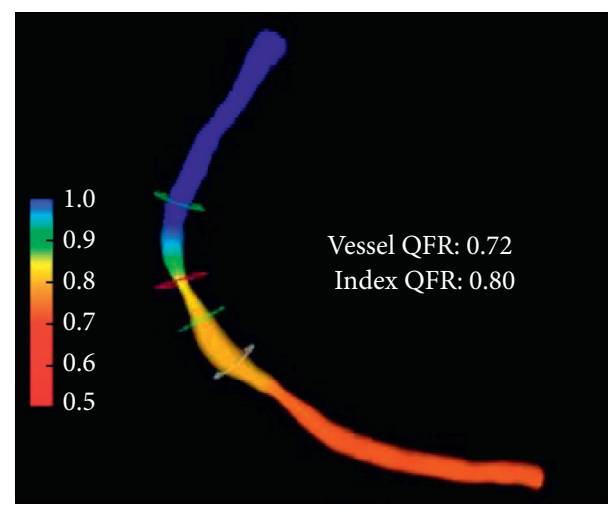

(b)

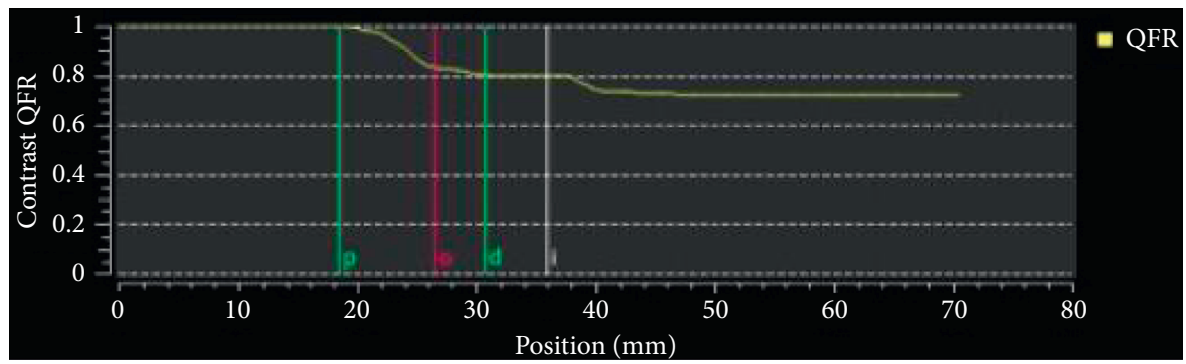

(c)

Figure 2: Haemodynamically significant intermediate stenosis assessed by QFR. (a) Coronary angiography showing a moderate lesion in the midright coronary artery with semiautomatic detection of lumen and vessel contours. The invasive FFR of this lesion showed haemodynamic significance $(F F R=0.73)$. (b) The computed colour-coded flow map of the reconstructed vessel. (c) Contrast-QFR pullback showing the pressure drop across the stenosis (p: proximal lesion marker; o: maximal lesion; d: distal lesion marker).

aQFR computation. Both cQFR and aQFR use patientspecific flow derived from frame count, which proved to be superior to fQFR when validated [17]. Over the recent years, several QFR studies have been validated against invasive FFR showing good correlation and diagnostic accuracy [16-19]. QFR is the first commercially available software to be CE-marked and FDA-cleared.

\subsection{Fractional Flow Reserve Derived from Coronary Angi-} ography (FFRangio), CathWorks Ltd, Kfar-Saba, Israel. FFRangio provides functional angiography mapping of the coronary tree with superimposed colour-coded FFR values. FFRangio's computational method is based on rapid flow analysis [20]. The 3D coronary tree, including its centreline, cross-sections at each point along it path, and exact topology, is automatically created based on the geometry of at least 3 angiographic projections. The next step involves the user validation process where the $3 \mathrm{D}$ shape of the coronary arteries is projected back onto the $2 \mathrm{D}$ angiographic images for inspection. Following this, the software applies a compensation mechanism where it uses all the available projections to correct any $x / y / z$ displacements due to breathing or patient movements during coronary angiography image acquisitions.

The coronary tree is then surfaced using a triangular mesh to display a $3 \mathrm{D}$ coronary model. The software automatically looks for a stenosis by performing systematic segment, branch, and junction level analysis. The coronary arterial network is modelled as an electrical circuit with each segment acting as a resistor. The vessel diameter and length determine its resistance. Each vessel's flow is based on its impact on overall resistance depending on the coronary tree arrangement, which is then lumped into a $3 \mathrm{D}$ model. Finally, haemodynamic evaluation of the stenosis is performed by considering the contribution of each narrowing to the total resistance and flow, which is then displayed as a colour-mapped coronary tree with FFR values at every stenosis. FFRangio is based on limited user interaction to reduce any discrepancy introduced by the user during processing.

The CathWorks FFRangio system is FDA-cleared and commercially available, with the unique advantage of providing reconstruction of the entire coronary tree with FFR values along each vessel.

\subsection{Simplified Model of FFR Calculation $\left(F F R_{\text {sim }}\right)$.} $\mathrm{FFR}_{\text {sim }}$ is an angiography-derived FFR methodology that is based on 3D-QCA and classic fluid dynamics equations without using finite element analysis [21]. The coronary angiography images are used to create a $3 \mathrm{D}$ angiographic reconstruction offline using a validated, commercially available software (QAngio XA Research Edition, 1.0, Medis specials bv, Leiden, the Netherlands). FFR sim $_{\text {calculation is }}$ based on the following simplified equation: 


$$
\begin{aligned}
\Delta P & =R * Q, \\
\Delta P_{\text {laminarflow }}= & \left(\frac{8 * \pi * n * L}{A^{2}}\right) * Q, \\
\Delta P_{\text {flowseperation }}= & k_{\text {flowseperation }} * \frac{P}{0.266} \\
& *\left(\frac{1}{\text { MLA }}-\frac{1}{\text { Adistal }}\right)^{2} * Q^{2},
\end{aligned}
$$

where, $k_{\text {flowseperation }}=1.21+0.08 *\left(\frac{L_{\text {lesion }}}{2 * \text { Dref.lesion }}\right)$,

where $\Delta P=$ pressure gradient; $R=$ resistance; $Q=$ volumetric flow; $n=$ blood viscosity; $L=$ lesion length; and $p=$ blood density.

Blood flow velocities are assumed identical proximal and distal to the stenosis at peak vasodilatation. The contrast flow along the vessel is calculated using the established TIMI frame count method. The flow rate in the main vessel will decrease proportionally to the cross-sectional area of the distal reference segment following a side branch. The distal mean arterial pressure $\left(P_{\mathrm{d}}\right)$ is calculated by subtracting the pressure drop across the lesion (derived from the above equations) from the mean arterial pressure $\left(P_{\mathrm{a}}\right)$. Finally, this allows calculation of $\mathrm{FFR}_{\text {sim }}$ which is defined as $\mathrm{Pd} / \mathrm{Pa}$ across as stenosis. Tar et al. studied 68 vessels with single-vessel stenosis where $\mathrm{FFR}_{\text {sim }}$ shows strong correlation with invasive FFR $(r=0.86, p<0.0001)$ [21]. The sensitivity and AUC of $\mathrm{FFR}_{\text {sim }}$ to detect haemodynamically significant lesions $($ FFR $<0.80)$ were 0.90 and 0.96 , respectively.

2.6. Cardiovascular Angiographic Analysis Systems for Vessel Fractional Flow Reserve (CAAS- vFFR), Pie Medical Imaging, Maastricht, The Netherlands. The CAAS-vFFR is an angiography-derived FFR software (Figure 3) based on the reconstruction of the coronary artery anatomy from two orthogonal coronary angiography views (at least $30^{\circ}$ difference in rotation/angulation) using the CAAS workstation [22]. ECG triggering, with an option for manual frame selection if needed, automatically performs temporal alignment of the cardiac cycle. Vessel contour detection is semiautomatic allowing for manual correction when necessary. The pressure drop calculation is instantaneous by assuming application of the physical law in coronary flow. The maximal hyperaemic blood flow in the proximal coronary artery is assumed to be preserved along the coronary of interest. The vFFR system calculates the pressure drop across a lesion based on the coronary flow behaviour physical laws, described by Gould [23] and Kirkeeide, but no further specific description of the methodology has been provided. Patient-specific aortic pressure is used during the analysis.

The FAST study showed that CAAS-vFFR had a diagnostic accuracy of $0.93(p<0.001)$ to detect lesions with FFR $<0.80$. A good linear correlation between FFR and vFFR $(r=0.89, p<0.001)$ was noted along with good reproducibility (interobserver variability $r=0.95, p<0.001$ ) [22]. The
CAAS-vFFR is CE-marked in Europe, FDA-cleared, and commercially available for clinical use.

\section{Discussion}

Over the last decade, clinical studies have demonstrated the feasibility and reproducibility of angiography-derived FFR software. These new technologies appear to correlate well with invasive FFR showing excellent diagnostic accuracy when tested offline (Table 1). However, there are significant differences in methodology, assumptions, and automation between these technologies. The advantages and limitations of the angiography-derived FFR software are summarised in Table 2.

The angiography-derived FFR can be obtained from either blood flow simulation using CFD or by mathematical formula. A recent meta-analysis found no difference between the two approaches for the diagnostic accuracy of angiography-derived FFR [26]. The successful application of CFD to CTCA images to predict blood flow and lesionspecific FFR preceded the development of angiographyderived FFR software. This prompted the studies of Morris et al., Papafaklis et al., and Tu et al., the first 3 angiographyderived FFR studies to apply CFD to the reconstructed coronary anatomical model for FFR prediction. The ability of angiography-derived software to predict FFR is novel with higher accuracy than reported for CTCA-based methodology. This has to be at least partially attributed to the superior resolution of invasive coronary angiography compared to computed tomography. The CFD approach is based on solving the fundamental governing equations of fluid dynamics-the continuity, momentum, and energy equations. The complex CFD modelling process requires prolonged computation time which has been a challenge to overcome for clinical applications. The first VIRTU-1 study by Morris et al. using transient (pulsatile) flow conditions required 24 hours of computing time for each vessel analysed. Subsequently, Tu et al. applied a steady-state flow simulation by assuming that the average pressure distribution over a cardiac cycle is no difference between these two approaches. This reduced computational time to 5 minutes per vessel representing a breakthrough in the field.

The complexity and time-consuming process of CFDbased methodology allowed the development of mathematical formula-based methodology. This allowed easier and faster calculation of pressure drop across a coronary stenosis. Two years following their initial study, Tu et al. developed a new methodology that avoided solving the traditional Navier-Stokes equation. QFR calculates the pressure drop across a stenosis using a simple quadratic equation using coefficients derived from flow data from their previous experimental models [17]. The instantaneous FFR computation meant that mathematical-based technologies became popular although subsequent studies applied a variety of different mathematical formulas. The CAAS-vFFR software calculates pressure drop across a stenosis by applying physical laws of coronary flow by Lance Gould and Kirkeeide. Both studies have reported good correlation and diagnostic accuracy compared to invasive FFR although 


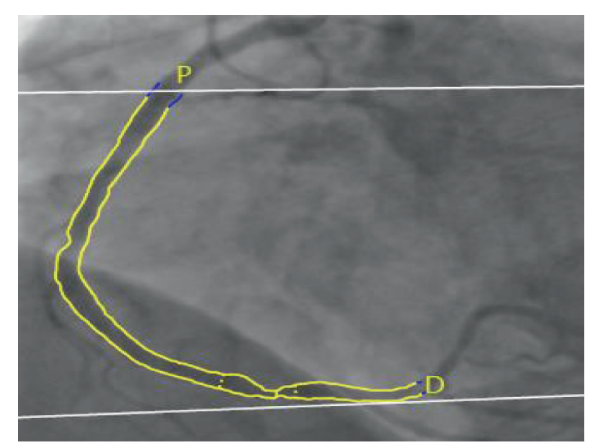

(a)

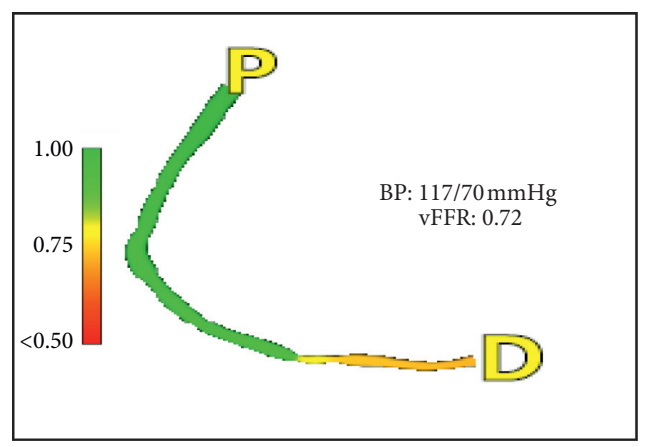

(b)

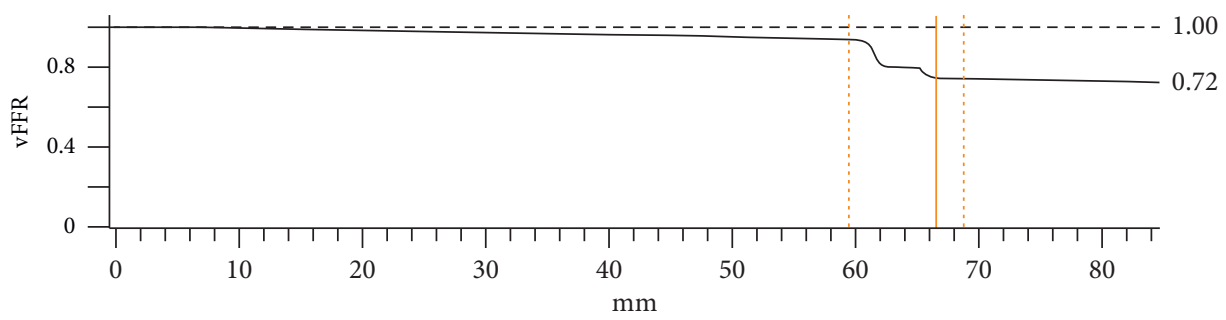

(c)

FIgURE 3: Haemodynamically significant intermediate stenosis assessed by CAAS-vFFR. (a) Coronary angiography demonstrating a moderate lesion in the distal right coronary artery with semiautomatic lumen border detection. The invasive FFR on this occasion is functionally significant $(\mathrm{FFR}=0.74)$. (b) Colour-coded pressure drop map of the reconstructed 3D model. Aortic pressure acquired during coronary angiography $(117 / 70 \mathrm{mmHg})$ is used for computation. (c) CAAS-vFFR pressure drop across the vessel highlighting the haemodynamic significance of the lesion.

there has no comparison between the different formula applications.

Segmentation is a key process of accurate 3D reconstruction. Most of the angiography-derived FFR software packages require just 2 projections acquired from routine coronary angiography. Biplane angiography is ideal but is not readily available in most catheterisation laboratories. However, most software try to correct for patient movement between angiography image acquisitions. The vFFR software is unique where it requires rotational coronary angiography, which offers multiple views to select the ideal projections for $3 \mathrm{D}$ reconstruction. However, rotational angiography is not widely used in clinical practice.

The inclusion of side branches as part of the 3D reconstruction has benefits and limitations. FFRangio provides a $3 \mathrm{D}$ reconstruction of the entire coronary tree, including side branches, with FFR values along each vessel. This is an attractive feature as it helps cardiologists plan individualised treatments, especially for patients with multivessel disease. The initial study by Tu et al. in 2014 included side branches as part of the 3D reconstruction. However, subsequently, the same group evolved the QFR software to focus on reconstruction of the main vessel only to significantly reduce the complexity and computation time. The authors concluded that the tapering reference diameter from 3D-QCA data can predict the decreasing mass flow rate along the vessel as the side branches are taking off while the mean flow velocity remains constant.

Most, if not all of the above-described methodologies are based on significant assumptions. Poiseuille law assumes a laminar flow travelling through a circular tube with constant cross-sectional area where the fluid is incompressible and Newtonian. Blood is non-Newtonian and the cross-sectional area of coronary arteries changes, even in a healthy vessel from proximal to distal end of the vessel. Despite these assumptions, the FFRangio showed good diagnostic accuracy when compared with invasive FFR. It is worth noting that FFRangio calculates the maximal flow rate in the stenosis compared to maximal flow rate in the absence of stenosis where else invasive FFR and several other angiography-derived models have focussed on the pressure gradients across a stenosis.

The coronary microcirculation, resistance, and functional capacity of the distal myocardial bed have a significant influence on coronary flow during hyperaemia [27]. vFAI is based on the fixed universal hyperaemic flow limit of $4 \mathrm{mls} /$ sec which is an oversimplification of complex boundary conditions. Myocardial damage following myocardial infarction, presence of collateral vessels, and raised myocardial resistance due to chronic conditions such as diabetes mellitus and hypertension can make vFAI assessments tricky and potentially inaccurate. A recent study of 300 patients showed that, in patients with coronary microvascular dysfunction, defined as high index of microcirculatory resistance (IMR) of $>23$ units, the positive predictive value of QFR significantly decreases from $93 \%$ to $67 \%$ [28]. This is likely due to microcirculatory involvement which is known to carry a higher risk of adverse outcomes [29]. Furthermore, QFR calculates pressure gradients by measuring flow whereas FFR estimates flow by measuring pressure 


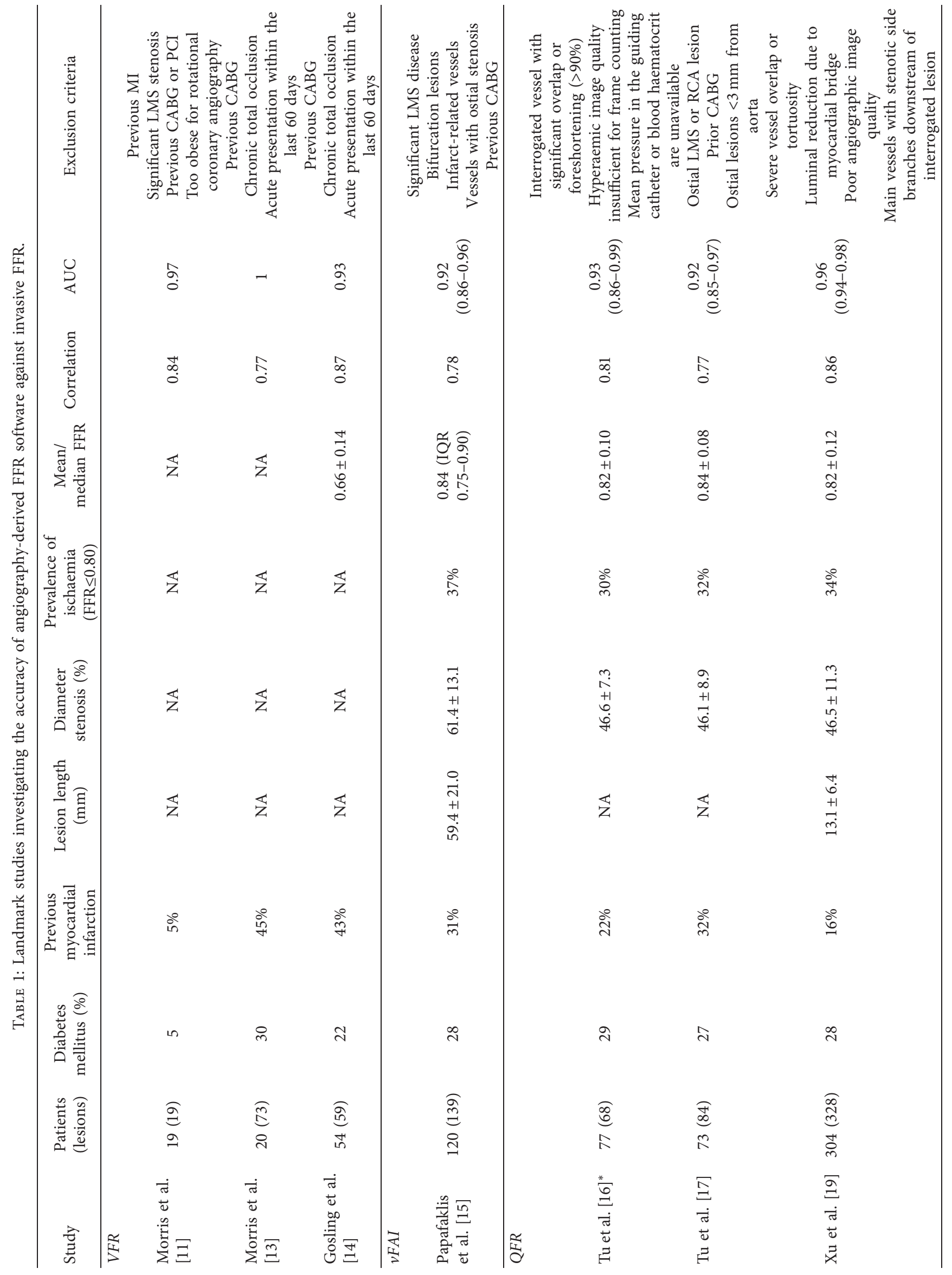




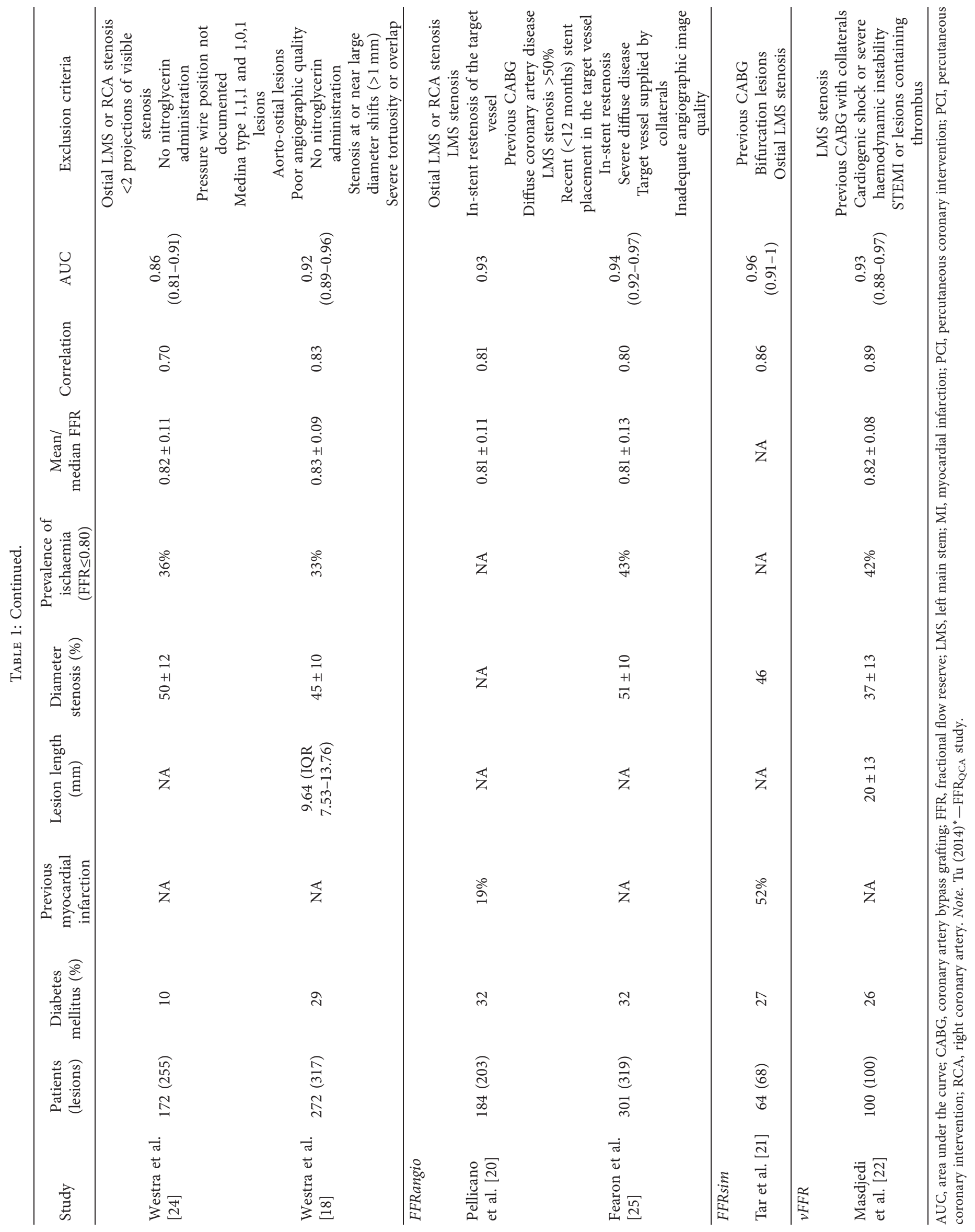


TABLE 2: Key advantages and limitations of all the angiography-derived FFR software.

\begin{tabular}{|c|c|c|}
\hline $\begin{array}{l}\text { Angiography-derived FFR } \\
\text { methodologies }\end{array}$ & Advantages & Limitations \\
\hline vFFR & $\begin{array}{c}\text { Fast FFR computation* } \\
\text { Incorporation of coronary microvascular information }\end{array}$ & $\begin{array}{l}\text { Need for rotational coronary angiography } \\
\text { Generic boundary condition }\end{array}$ \\
\hline vFAI & $\begin{array}{l}\text { First methodology with a reasonable computation time } \\
\text { with a clinical potential }\end{array}$ & $\begin{array}{c}\text { Single-vessel analysis } \\
\text { Assumption of static coronary flow across the } \\
\text { vessel }\end{array}$ \\
\hline QFR & $\begin{array}{c}\text { Instantaneous FFR computation } \\
\text { User-friendly interface } \\
\text { Estimates flow from patient-specific data and TIMI } \\
\text { frame count } \\
\text { Extensively validated against FFR }\end{array}$ & $\begin{array}{l}\text { Single vessel analysis } \\
\text { Unsuitable for aorto-ostial lesion assessment } \\
\text { Need for nitroglycerin administration prior to } \\
\text { angiography image acquisition }\end{array}$ \\
\hline FFRangio & $\begin{array}{l}\text { Complete functional assessment of coronary tree } \\
\text { Fast FFR computation } \\
\text { Able to reconstruct coronary artery anatomy using } \\
\text { more than } 2 \text { angiographic projections }\end{array}$ & Unsuitable for aorto-ostial lesion assessment \\
\hline FFRsim & $\begin{array}{c}\text { Simplified equation for FFR calculation } \\
\text { Flow distribution before and after bifurcation was } \\
\text { calculated }\end{array}$ & $\begin{array}{c}\text { Single validation study } \\
\text { TIMI frame count used following administration } \\
\text { of intracoronary adenosine }\end{array}$ \\
\hline CAAS-vFFR & $\begin{array}{l}\text { Instantaneous FFR computation } \\
\text { User-friendly interface }\end{array}$ & $\begin{array}{c}\text { Single-vessel analysis } \\
\text { Unsuitable for aorto-ostial lesion assessment } \\
\text { Need for invasive blood pressure information }\end{array}$ \\
\hline
\end{tabular}

${ }^{*}$ Fast FFR computation is based on the latest VIRTU-Fast study [13].

gradients. Despite this, the negative predictive value of the patients with high IMR in this study remained high at $87 \%$ highlighting the value of QFR in identifying low-risk patients.

Similarly, because vFFR assumes standardized downstream resistance, $\mathrm{vFFR}$ values are likely to underestimate the significance of a coronary stenosis in cases where the microcirculatory resistance in the distal coronary bed is elevated. The fQFR model of QFR relies on a hyperaemic fixed velocity of $0.35 \mathrm{~m} / \mathrm{s}$, irrespective of the coronary vessels. This is in contrast to some reports where the left anterior descending artery flow was noted to be significantly higher $(0.62 \mathrm{~m} / \mathrm{s})$ than other vessels [30]. Given the flow and pressure relationship of the quadratic equation, it is not surprising that the CQFR and aQFR both showed better correlation to invasive FFR when compared to fQFR. Additionally, there may be a difference between coronary flow and perfusion between the left and right coronary arteries due to the systolic pressure differences between the ventricles [22]. This may be relevant for QFR analysis, specifically CQFR and aQFR where the frame counting is key during the computation of QFR values. However, no information is available detailing this potential pitfall in the published QFR literature.

\section{Conclusion}

Several angiography-derived FFR methodologies have shown good correlation and accuracy with invasive FFR to detect haemodynamically significant lesions, independent of CFD-based or simplified mathematical approaches. All methods have some variation in their assumptions for computing FFR values. At present, 3 angiography-derived
FFR software packages (QFR, CAAS-vFFR, and FFRangio) are commercially available, based on offline validation studies that demonstrate fast and reliable 3D reconstruction and FFR computation. Clinical outcomes studies are currently underway (FAVOR III EJ: NCT03729739 and FAVOR III China: NCT03656848) and highly anticipated to incorporate angiography-derived FFR technologies in our daily clinical decision-making and guidelines. This will revolutionise our interpretation of diagnostic angiography by improving patient risk stratification through greater penetration in clinical practice and ultimately improve patient outcomes.

\section{Conflicts of Interest}

The authors declare that they have no conflicts of interest.

\section{Acknowledgments}

This research and $\mathrm{AR}, \mathrm{RB}, \mathrm{AM}, \mathrm{CB}$, and $\mathrm{AB}$ were funded by Barts NIHR Biomedical Research Centre, London, UK.

\section{References}

[1] F. J. Neumann, M. Sousa-Uva, A. Ahlsson et al., "2018 ESC/ EACTS guidelines on myocardial revascularization," European Heart Journal, vol. 40, pp. 87-165, 2019.

[2] S.-J. Park, S.-J. Kang, J.-M. Ahn et al., "Visual-functional mismatch between coronary angiography and fractional flow reserve," JACC: Cardiovascular Interventions, vol. 5, no. 10, pp. 1029-1036, 2012.

[3] M. Safi, V. Eslami, M. H. Namazi et al., "Visual-functional mismatch between coronary angiography, fractional flow reserve, and quantitative coronary angiography," The 
International Journal of Angiology, vol. 25, no. 4, pp. 229-234, 2016.

[4] H. V. Anderson, G. S. Roubin, P. P. Leimgruber et al., "Measurement of transstenotic pressure gradient during percutaneous transluminal coronary angioplasty," Circulation, vol. 73, no. 6, pp. 1223-1230, 1986.

[5] N. H. J. Pijls, B. De Bruyne, K. Peels et al., "Measurement of fractional flow reserve to assess the functional severity of coronary-artery stenoses," New England Journal of Medicine, vol. 334, no. 26, pp. 1703-1708, 1996.

[6] G. J. W. Bech, B. De Bruyne, N. H. J. Pijls et al., "Fractional flow reserve to determine the appropriateness of angioplasty in moderate coronary stenosis," Circulation, vol. 103, no. 24, pp. 2928-2934, 2001.

[7] P. A. L. Tonino, B. De Bruyne, N. H. J. Pijls et al., "Fractional flow reserve versus angiography for guiding percutaneous coronary intervention," New England Journal of Medicine, vol. 360, no. 3, pp. 213-224, 2009.

[8] B. De Bruyne, N. H. J. Pijls, B. Kalesan et al., "Fractional flow reserve-guided PCI versus medical therapy in stable coronary disease," New England Journal of Medicine, vol. 367, no. 11, pp. 991-1001, 2012.

[9] A. S. C. Yong, A. C. C. Ng, D. Brieger, H. C. Lowe, M. K. C. Ng, and L. Kritharides, "Three-dimensional and twodimensional quantitative coronary angiography, and their prediction of reduced fractional flow reserve," European Heart Journal, vol. 32, no. 3, pp. 345-353, 2011.

[10] C. V. Bourantas, A. C. Tweddel, M. I. Papafaklis et al., "Comparison of quantitative coronary angiography with intracoronary ultrasound. Can quantitative coronary angiography accurately estimate the severity of a luminal stenosis?" Angiology, vol. 60, no. 2, pp. 169-179, 2009.

[11] P. D. Morris, D. Ryan, A. C. Morton et al., "Virtual fractional flow reserve from coronary angiography: modeling the significance of coronary lesions," JACC: Cardiovascular Interventions, vol. 6, no. 2, pp. 149-157, 2013.

[12] N. Westerhof, J.-W. Lankhaar, and B. E. Westerhof, "The arterial Windkessel," Medical \& Biological Engineering \& Computing, vol. 47, no. 2, pp. 131-141, 2009.

[13] P. D. Morris, D. A. Silva Soto, J. F. A. Feher et al., "Fast virtual fractional flow reserve based upon steady-state computational fluid dynamics analysis," JACC: Basic to Translational Science, vol. 2, no. 4, pp. 434-446, 2017.

[14] R. C. Gosling, P. D. Morris, D. A. Silva Soto, P. V. Lawford, D. R. Hose, and J. P. Gunn, "Virtual coronary intervention," JACC: Cardiovascular Imaging, vol. 12, no. 5, pp. 865-872, 2019.

[15] M. I. Papafaklis, T. Muramatsu, Y. Ishibashi et al., "Fast virtual functional assessment of intermediate coronary lesions using routine angiographic data and blood flow simulation in humans: comparison with pressure wire - fractional flow reserve," EuroIntervention, vol. 10, no. 5, pp. 574-583, 2014.

[16] S. Tu, E. Barbato, Z. Köszegi et al., "Fractional flow reserve calculation from 3-dimensional quantitative coronary angiography and TIMI frame count," JACC: Cardiovascular Interventions, vol. 7, no. 7, pp. 768-777, 2014.

[17] S. Tu, J. Westra, J. Yang et al., "Diagnostic accuracy of fast computational approaches to derive fractional flow reserve from diagnostic coronary angiography," JACC: Cardiovascular Interventions, vol. 9, no. 19, pp. 2024-2035, 2016.

[18] J. Westra, B. K. Andersen, G. Campo et al., "Diagnostic performance of in-procedure angiography-derived quantitative flow reserve compared to pressure-derived fractional flow reserve: the FAVOR II Europe-Japan study," Journal of the American Heart Association, vol. 7, 2018.
[19] B. Xu, S. Tu, S. Qiao et al., "Diagnostic accuracy of angiography-based quantitative flow ratio measurements for online assessment of coronary stenosis," Journal of the American College of Cardiology, vol. 70, no. 25, pp. 3077-3087, 2017.

[20] M. Pellicano, I. Lavi, B. De Bruyne et al., "Validation study of image-based fractional flow reserve during coronary angiography," Circulation Cardiovascular Interventions, vol. 10, 2017.

[21] B. Tar, C. Jenei, C. A. Dezsi et al., "Less invasive fractional flow reserve measurement from 3-dimensional quantitative coronary angiography and classic fluid dynamic equations," EuroIntervention, vol. 14, no. 8, pp. 942-950, 2018.

[22] K. Masdjedi, L. J. C. van Zandvoort, M. M. Balbi et al., "Validation of 3-dimensional quantitative coronary angiography based software to calculate fractional flow reserve: fast assessment of STenosis severity (FAST)-study," EuroIntervention, 2019.

[23] K. L. Gould, K. O. Kelley, and E. L. Bolson, "Experimental validation of quantitative coronary arteriography for determining pressure-flow characteristics of coronary stenosis," Circulation, vol. 66, no. 5, pp. 930-937, 1982.

[24] J. Westra, S. Tu, S. Winther et al., "Evaluation of coronary artery stenosis by quantitative flow ratio during invasive coronary angiography: the WIFI II study (Wire-Free functional imaging II)," Circulation Cardiovascular Imaging, vol. 11, Article ID e007107, 2018.

[25] W. F. Fearon, S. Achenbach, T. Engstrom et al., "Accuracy of fractional flow reserve derived from coronary angiography," Circulation, vol. 139, no. 4, pp. 477-484, 2019.

[26] C. Collet, Y. Onuma, J. Sonck et al., "Diagnostic performance of angiography-derived fractional flow reserve: a systematic review and Bayesian meta-analysis," European Heart Journal, vol. 39, no. 35, pp. 3314-3321, 2018.

[27] M. J. Kern, A. Lerman, J.-W. Bech et al., "Physiological assessment of coronary artery disease in the cardiac catheterization laboratory," Circulation, vol. 114, no. 12, pp. 1321-1341, 2006.

[28] H. Mejía-Rentería, J. M. Lee, F. Lauri et al., "Influence of microcirculatory dysfunction on angiography-based functional assessment of coronary stenoses," JACC: Cardiovascular Interventions, vol. 11, no. 8, pp. 741-753, 2018.

[29] T. P. van de Hoef, M. A. van Lavieren, P. Damman et al., "Physiological basis and long-term clinical outcome of discordance between fractional flow reserve and coronary flow velocity reserve in coronary stenoses of intermediate severity," Circulation: Cardiovascular Interventions, vol. 7, no. 3, pp. 301-311, 2014.

[30] E. O. Ofili, M. J. Kern, J. A. St Vrain et al., "Differential characterization of blood flow, velocity, and vascular resistance between proximal and distal normal epicardial human coronary arteries: analysis by intracoronary Doppler spectral flow velocity," American Heart Journal, vol. 130, no. 1, pp. 37-46, 1995. 